This item was submitted to Loughborough's Research Repository by the author.

Items in Figshare are protected by copyright, with all rights reserved, unless otherwise indicated.

\title{
Ontology mapping using description logic and bridging axioms
}

PLEASE CITE THE PUBLISHED VERSION

http://dx.doi.org/10.1016/j.compind.2012.09.004

\section{PUBLISHER}

(c) Elsevier

\section{VERSION}

AM (Accepted Manuscript)

\section{PUBLISHER STATEMENT}

This work is made available according to the conditions of the Creative Commons Attribution-NonCommercialNoDerivatives 4.0 International (CC BY-NC-ND 4.0) licence. Full details of this licence are available at: https://creativecommons.org/licenses/by-nc-nd/4.0/

\section{LICENCE}

CC BY-NC-ND 4.0

\section{REPOSITORY RECORD}

Kumar, Sri K., and Jennifer A. Harding. 2019. “Ontology Mapping Using Description Logic and Bridging Axioms". figshare. https://hdl.handle.net/2134/20538. 


\title{
Ontology Mapping Using Description Logic and Bridging Axioms
}

\author{
Sri Krishna Kumar* and Dr. J. Harding** \\ ${ }^{*}$ Wolfson School of Mechanical Engineering, Loughborough University, UK \\ **Communicating Author: J.A.Harding@lboro.ac.uk
}

\begin{abstract}
:
In the last decade various proposals have been made to promote fruitful and efficient collaboration among small and medium sized enterprises (SMEs) in the form of virtual enterprises (VEs). VEs are opportunity driven temporary collaborations of heterogeneous enterprises and their success depends on seamless interoperability of knowledge and data sharing. Ontology adoption or implementation is becoming an essential and successful tool for VE operation but commonly ontology mapping is also required to achieve interoperability. The current state of the art in ontology mapping indicates that mapping systems require a great deal of human intervention as the initial merging of the ontologies brings various types of conflicts and inconsistencies. The ontology mapping method proposed in this paper uses description logic (DL) based bridging axioms between the ontologies. Atomic concept level similarity has been taken as input to establish the complex concepts and roles level mapping. A manufacturing enterprise ontology and a marketing enterprise ontology are considered and their mapping has been demonstrated as an example of the proposed mapping process.
\end{abstract}

Keywords: Ontology mapping, Description Logic, Bridging Axioms, Virtual Enterprise

\section{Introduction:}

Today, enterprises are facing a rapidly changing business environment characterized by globalization, disruptive market conditions, fast technological transformations etc. In this scenario, business competition and survival have shifted from individual enterprise level considerations to value chain (collaborative level) challenges and networking, cooperation, outsourcing and customization must be examined as potential solutions for enterprise survival. Enterprises are focused on enhancing their competitive performance through efficient and effective linkage of both internal and external 
operations. Moreover, to achieve the best results from their limited resources, SMEs commonly concentrate closely on their core competencies whilst outsourcing or collaborating with other enterprises for complementary capability, operation etc. This results in collaborating networks of enterprises which have been classified as Extended enterprises (EE) or Virtual enterprises (VE) (Jagdev and Thoben 2001), which exist purely for the duration of the collaboration (Martinez et al. 2001).

A VE, which is a temporary network of enterprises, is created for the realization of a business opportunity and is dissolved after achieving that business opportunity. It is commonly argued that participating in VEs is a key aspect for surviving in the competitive business environment. In a VE, enterprises need to communicate, cooperate, collaborate and interoperate with other member enterprises situated locally or globally. Thus, the essential requirements for effective and efficient collaboration among enterprises are agility and interoperability (Chen et. al. 2008). Information and communication technology (ICT) enables enterprises to transfer data and information at a technical level, but does not provide interoperability at the semantic level.

VEs need to address many issues related to interoperability, including the most important issue of semantic interoperability. Semantic interoperability ensures that the intention and meaning of information transferred between the enterprises will be understood correctly. In order to achieve semantic interoperability enterprises may use ontology based information, data and knowledge acquisition and transfer (see Gruber 1995 for a definition of ontology). Enterprises will develop their ontologies based on individual requirements (manufacturing, logistics etc.) and may use different terminologies for the concepts. This has led to another kind of heterogeneity, semantic heterogeneity, which still remains a key issue for VE interoperability.

The use of ontologies facilitates the representation of shared concepts in a domain or across domains by specifying a set of terms to ensure proper communication between the enterprises. Ontologies explicitly represent the data along with their semantics to facilitate correct information transfer; however, it is still very difficult as yet to develop a single, universally accepted, ontology, defining the whole universal system (Pollalis and Dimitriou, 2008). At present, different organizations are developing their own ontologies, in most cases independently, to describe the same, different or overlapping 
domains. In order to achieve proper semantic interoperability, ontologies need to be synchronized through ontology mapping and matching. Ontology mapping finds the correlation between entities (concept, relation, individuals) among the different enterprises. In literature, various approaches have been reported for ontology mapping (sometimes referred to as alignment) (Chungoora and Young 2008) ranging from manual (Hu et. al. 2008) to semi-automatic (Chen et al. 2011). This has generated different forms of heterogeneity among the ontologies known as lexical heterogeneity (same concept defined by different terms or the same terms being used to define different concepts), structural heterogeneity (difference in degree of details or granularity) etc. Various heterogeneities have been reported in ontology mapping (Wang and Liu, 2009) and the reported types of mismatches are as follow:

\section{Conflicts in ontology mapping:}

1. Synonymy conflicts: Same concepts defined by different terms.

2. Polysemy conflicts: Different terms defined by the same term.

3. Subclass conflicts: Occur when the same class in different ontologies is divided into different subclass concepts (i.e. difference in the granularity).

4. Class-Role conflicts: Occurs when a class in one ontology is described by a role or properties in another ontology.

5. Class Coverage conflicts: Occurs when a class defines the same concept in two ontologies but one class covers a broader domain than the other.

6. Role conflicts: Occurs when the same class in different ontologies is described by different properties (roles).

7. Role Attribute conflicts: Occurs when a class and its role are the same in two ontologies but their value types (attributes) differ.

The mismatches in the ontology mapping are due to assigning simple correspondence between entities which creates an erroneous or inconsistent mapping (Dou and McDermott, 2006). Figure 1 shows an example in which two different ontologies have been mapped, assuming consistent ontologies, simple similarity measures between them will provide the following correspondence:

01: Operation $\rightarrow$ 02: Operation (i),

01: Transportation $\rightarrow$ 02: Transportation (ii). 
Using the sub-concept relationship in the ontology 01 , the following can be established:

01: Transportation $\rightarrow$ 01: Operation

Using equations (i), (ii) and (iii), following can be inferred:

02: Transportation $\rightarrow$ 02: Operation, but this is incorrect as they are disjoint concepts. Hence, according to the mapping, Transportation can be inferred as a sub-concept of the Operation in 02, therefore a logical inconsistency has occurred and therefore the mapping becomes erroneous. Such inconsistencies in mapping not only exist at the concept level but also at the role level. Differences may also exist at the level of granularity, which can be demonstrated by considering further detail of a product specification in two ontologies. This for example might be given as: 01: hasBore $(\mathrm{x}, \mathrm{y})$ and 02: hasDiameter $(\mathrm{x}, \mathrm{y})$, which means product $\mathrm{x}$ has bore (or diameter) $\mathrm{y}$. Translating or mapping the role from 01 to 02 gives hasBore(x,y) $\rightarrow$ hasDiameter(x,y). This mapping is consistent but referring back to 01 from 02 , hasDiameter $(\mathrm{x}, \mathrm{y}) \rightarrow$ hasBore(x,y) may be inconsistent because anything having a diameter does not necessarily imply having a bore.

\section{$<<$ Insert Figure 1 about here $>>$}

As shown in the above examples, the simple correspondence between the terms of two ontologies commonly causes inference problems. This can be explained as: assuming P, $\mathrm{Q}$ are two terms in two ontologies, with simple correspondence $P \rightarrow Q$ then $\{\mathrm{KB}, \mathrm{P}$, $P \rightarrow Q\} \vdash \quad Q$ i.e. $Q$ can be inferred, but $\{\mathrm{KB}, \mathrm{P}, P \rightarrow Q\} \Vdash \neg P \rightarrow \neg Q$, i.e. $\{\mathrm{KB}, \mathrm{P}$, $P \rightarrow Q\} \forall Q \rightarrow P$.In general terms the fact that the $Q$ can be inferred from $P$ does not automatically mean that $P$ can be inferred from $Q$ and trying to infer this causes the error in the ontology mapping as described earlier. Such heterogeneity in the ontology mapping is caused by considering the correspondence but not analysing their relationship, such as more general $(\supseteq)$, less general $(\subseteq)$, equivalence $(\equiv)$, disjoint $(\perp)$, overlapping $(\cap)$ or union of other entities $(\mathrm{e} 1 \cup \mathrm{e} 2 \cup \ldots . .$.$) . Defining such relationships$ in the mapping can prevent incorrect inferexce, For example if the mapping finds a correspondence such as $P \subseteq Q$, this gives the KB two axioms: $P \rightarrow Q$ and $\neg P \rightarrow \neg Q$ (not $\mathrm{P}$ does not imply not $\mathrm{Q}$ ), for example hasBore(x,y) $\subseteq$ hasDiameter(x,y) mapping with defining relationship will not infer hasDiameter $(x, y) \rightarrow$ hasBore $(x, y)$ whereas 
considering only the correspondence between the terms will produce the wrong inference.

The rest of this paper is organized as follows: a literature review has been presented in the next section. Section 3 introduces the concept of description logic (DL). Logical derivation of bridging axioms between the ontologies is explained in section 4 . The implementation method, with an example, is presented in section 5 . This paper concludes in the section 6 with a discussion of future research areas.

\section{Ontology Mapping related works:}

Considerable effort has been put into ontology mapping research in order to provide interoperability and resolve conflicts (as described in the previous section). A comprehensive review of current approaches in ontology mapping has been provided by Euzenat and Shvaiko (2007). Initial work on ontology mapping was focused mainly on the string distance and overall nomenclature of the ontologies. This approach commonly leads to synonymy and polysemy conflicts as cross domain ontologies or even similar domain ontologies often use different taxonomies. Ontology mapping systems, such as FCA-merge (Stumme and Madche, 2001) and T- Tree (Euzenat, 1994) tried to resolve this issue and explore the subclass- superclass relationships along with lexical similarity for ontology mapping. Various other approaches have also been applied in ontology mapping and alignment as shown in Table 1.

$$
<<\text { Insert Table } 1 \text { about here }>>
$$

Nowadays, especially in the VE paradigm, enterprises can use OWL (web ontology language) as a prominent tool for storing, using and transferring data and knowledge through the web. OWL is based on the Description logic (DL), a fragment of first order logic (FOL). CtxMatch (Bouquet et al., 2006) and S-Match (Giunchiglia, 2004) tried to determine semantic matching with inconsistency deduction using the Description Logic (DL) axioms. In these approaches inconsistencies were detected using the unsatisfiability of the equivalence and sumsumption relation but still simple correlation between the terms may cause the heterogeneity even in the DL based ontology mapping (Dou and McDermott, 2006). 
Current literature indicates that there is a spectrum of these methods that rely on lexical similarity matching, which from a semantic interoperability viewpoint is not optimal. Moreover, where VEs are relying on the web for knowledge and data transfer, it becomes imperative to consider ontology mapping in the DL paradigm. This is because the web ontology OWL, is based on DL, hence concepts defined in different ontologies using OWL need to be brought under a single umbrella to achieve interoperability. The next section describes DL and the procedure adopted in this paper to achieve interoperability.

\section{Description Logic:}

Description logic (DL), a decidable fragment of first order logic (FOL), is the backbone of OWL. OWL was proposed by the World Wide Web Consortium (W3C) in order to develop the semantic web (W3C, 2000). A DL knowledge base, KBase, contains the explicit and implicit information about the ontology and is composed by the Terminological box, TBox (T) and Assertion box , ABox (A), which is denoted as Kbase $=\langle\mathrm{T}, \mathrm{A}\rangle$.

The Tbox defines the terminology i.e. the vocabulary of the domain, while the ABox contains the assertions about the individuals based on the terminology. The KBase building elements are atomic concepts and roles, which are self-explanatory terms and cannot be derived using other concepts and roles. Complex concepts and roles are built using atomic concepts, roles and DL constructors. From atomic roles and concepts (A, P), complex concepts and roles $(\mathrm{C}, \mathrm{R})$ are built using DL constructors.

The concepts $(C)$ are formed from the atomic concepts using top concept ( T), bottom concept $(\perp)$, negation $(\neg A)$, union $\left(C_{1} \sqcup C_{2}\right)$, intersection $\left(C_{1} \sqcap C_{2}\right)$, existential quantifier $(\exists R . C)$, universal quantifier $(\forall R . C)$, cardinality restriction $\left(\geq_{n}\right.$ R.C, $\leq_{n}$ R.C $)$ etc.

Roles (R) are constructed from atomic roles $(P)$, negation $(\neg R)$, transitive $\left(R^{+}\right)$, inverse roles $\left(R^{-}\right)$etc.

Concepts and roles, in DL are seen as unary and binary relations such as: $\mathrm{C}(\mathrm{x})$ and $\mathrm{R}(\mathrm{y}$, $\mathrm{z}$ ). In this, $\mathrm{x}$ satisfies the concept $\mathrm{C}$ and $\mathrm{y}$ and $\mathrm{z}$ are in relation $\mathrm{R}$. 


\section{Logical Derivation of Ontology Mapping and Bridging Axioms:}

Ontology mapping (sometimes called translation) is a difficult task especially across domains as in the case of a VE. Every enterprise develops its own terminology and axioms relating the terminology. In this research, ontology mapping is obtained by first merging the ontologies together by taking the union of terms and axioms and whilst preserving their namespace. Secondly bridging axioms are built between the terms in the two ontologies in order to build a global ontology which is then ready to merge with further ontologies. The reason for forming a global ontology lies in the fact that only $n-1$ mappings are required for $\mathrm{n}$ ontologies whereas, ${ }^{\mathrm{n}} \mathrm{C}_{2}$ mappings are required in the case of one to one mappings. Furthermore, any change in an ontology is easier to incorporate using a global ontology than one to one mapping.

The process of ontology mapping, proposed in this paper, has been depicted in the figure (2). Although, great effort has been put into achieving fully automatic ontology mapping, human intervention is still needed for the final verification. In this research, the developed mapping technique takes a step closer towards automation by reducing human mediation. As shown in the figure (2), human input is required for defining the relationships between atomic concepts in two ontologies (TBox input) or providing individuals and their roles as ABox input. The rest of the mapping is then carried out, automatically. DL based reasoning has been used, which verifies and validates the mapping to form a valid global ontology. The next section describes the DL based logical derivation for ontology mapping.

$<<$ Insert Figure 2 about here $>>$

\subsection{Process description:}

Ontology mapping through ontology merging and bridging axioms can be given by the following function: $f:\left\{O_{1}, O_{2} \ldots\right\} \rightarrow\left\{G O, B R_{i j}\right\}$. Where $O_{i}$ denotes the merging ontologies, $\mathrm{GO}$ denotes the global ontology and $\mathrm{BR}_{i j}$ is the set of rules (Bridging rules or axioms) inter-relating the entities of $\mathrm{O}_{i}$ and $\mathrm{O}_{j}$. The bridging rule $\mathrm{BR}_{i j}$ w.r.t. $\mathrm{O}_{i}$ and $\mathrm{O}_{j}$ is said to be consistent if the following equation holds: 
$\left\{K B_{G O} ; O_{i}(e)\right\} \vDash_{B R_{i j}} O_{j}(e)$ and $\left\{K B_{G O} ; O_{j}(e)\right\} \vDash_{B R_{j i}} O_{i}(e)$, where $K B_{G O}=\bigcup_{i}\left(T_{i} \cup A_{i}\right)$ is the union of TBoxes and ABoxes of the merging ontologies. The above equation ensures that the mapping should be consistent locally, i.e. mapping from $\mathrm{O}_{i}$ to $\mathrm{O}_{j}$ using $\mathrm{BR}_{i j}$ should be consistent w.r.t. $\mathrm{O}_{j}$.

In general, ontologies are described by concepts (unary relations) and their roles (binary relations between concepts) and therefore an ontology's interpretation $I$ consists of the non-empty set $\Delta^{I}$, the domain of interpretation. Every interpretation of a concept $C$ is a set $C^{I} \subseteq \Delta^{I}$ and every interpretation of role $\mathrm{R}$ is $R^{I} \subseteq \Delta^{I} \times \Delta^{I}$. Using the prefix $i$ and $j$ for respective ontologies, the derivations of bridging axioms between ontologies are as follows.

1. Concept level: At the concept level the bridging axiom $\mathrm{BR}_{i j}$ is the relation from $\Delta^{I_{i}}$ from $\Delta^{I_{j}}$ and is the subset of $\Delta^{I_{i}} \times \Delta^{I_{j}}$. The process of identifying relationships between the concepts of two ontologies can be obtained by identifying relationships between the atomic concepts of two ontologies. Atomic concepts are used to build the complex concepts. Many researchers use the jargon base concept for atomic concepts and name concept or defined concepts for complex concepts. This paper uses the term atomic concepts and complex concepts. The process of bridging at atomic concept level can be achieved either manually or by expert's interpretation i.e. manual entry of atomic concept relationships as bridging rules or by using the Abox queries as follows:

a. Equivalence relation: Atomic concepts A and B in two ontologies are said to be equivalent if the following holds: $\{G O, B R\} \vDash i: A \equiv j: B$ iff $\forall x, A(x) \leftrightarrow B(x)$ with the bridging rule $B R_{i j} \rightarrow i: A \equiv j: B$

b. Subsumption-Supersumption relation: Atomic concept A and B in two ontologies can be inferred as a Subsumption-Supersumption relation if the following holds:

$\{M O, B R\} \vDash i: A \subseteq j: B$ iff (i) $\forall x, A(x) \rightarrow B(x)$ and (ii) $\exists y, B(y) \wedge \neg A(y)$

with the bridging rule $B R_{i j} \rightarrow i: A \subseteq j: B$ 
c. Overlapping relation: Two atomic concepts A and B are in an overlapping relationship if the following holds:

$\{M O, B R\} \vDash(A \cap B)$ iff $\exists x, A(x) \wedge B(x)$ with the bridging rule $B R_{i j} \rightarrow i: A \cap j: B$

d. Disjoint relation: Two concepts are said to be in a disjoint relationship if the following holds:

$\{M O, B R\} \vDash(A \perp B)$ iff $\forall x, A(x) \leftrightarrow \neg B(x)$ with the bridging rule $B R_{i j} \rightarrow i: A \equiv j: \neg B$

For complex concepts, which are built from atomic concepts and roles, bridging axioms can be determined by analysing the atomic concepts and roles relationships. To illustrate the method applied in this research, consider the complex concepts $\mathrm{C}_{i}$ and $\mathrm{C}_{j}$ which are defined as:

$C_{i}=A_{1} \cap P . A_{2} \quad$ and $\quad C_{j}=B_{1} \cap R \cdot B_{2}$ where the concepts and roles in the definition are atomic ones. Now the bridging axioms can be determined as:

a. Equivalence relation: Concepts $\mathrm{C}_{i}$ and $\mathrm{C}_{j}$ as defined above, in two ontologies are said to be equivalent if the following holds:

$\{G O, B R\} \vDash i: C_{i} \equiv j: C_{j}$ iff $\left\{\left(A_{1} \equiv A_{2}\right) \wedge\left(B_{1} \equiv B_{2}\right) \wedge(P \equiv R)\right\}$

b. Subsumption-Supersumption relation: Concept $\mathrm{C}_{i}$ and $\mathrm{C}_{j}$ in two ontologies can be inferred to be in a Subsumption-Supersumption relationship if the following holds:

$$
\begin{aligned}
\{M O, B R\} \vDash i: C_{i} \subseteq j: C_{j} & \text { iff (i) }\left\{\left(A_{1} \equiv A_{2}\right) \wedge\left(B_{1} \equiv B_{2}\right) \wedge(P \subseteq R)\right\} \text { or } \\
& \text { (ii) }\left\{\left(A_{1} \equiv A_{2}\right) \wedge\left(B_{1} \subseteq B_{2}\right) \wedge(P \equiv R)\right\} \text { or } \\
& \text { (iii) }\left\{\left(A_{1} \subseteq A_{2}\right) \wedge\left(B_{1} \equiv B_{2}\right) \wedge(P \equiv R)\right\} \text { or } \\
& \text { (iv) }\left\{\left(A_{1} \subseteq A_{2}\right) \wedge\left(B_{1} \subseteq B_{2}\right) \wedge(P \equiv R)\right\} \text { or } \\
& \text { (v) }\left\{\left(A_{1} \subseteq A_{2}\right) \wedge\left(B_{1} \equiv B_{2}\right) \wedge(P \subseteq R)\right\} \text { or } \\
& \text { (vi) }\left\{\left(A_{1} \equiv A_{2}\right) \wedge\left(B_{1} \subseteq B_{2}\right) \wedge(P \subseteq R)\right\} \text { or } \\
& \text { (vii) }\left\{\left(A_{1} \subseteq A_{2}\right) \wedge\left(B_{1} \subseteq B_{2}\right) \wedge(P \subseteq R)\right\}
\end{aligned}
$$

c. Overlapping relation: Two concepts $\mathrm{C}_{i}$ and $\mathrm{C}_{j}$ are in an overlapping relationship if the following holds: $\{M O, B R\} \vDash\left(C_{i} \cap C_{j}\right)$ iff $\left\{\left(A_{1} \wedge B_{1}\right) \wedge\left(A_{2} \wedge B_{2}\right) \wedge(P \wedge R)\right\}$.

d. Disjoint relation: Two concepts $C_{i}$ and $C_{j}$ are said to be in disjoint relation if following holds:

e. $\{M O, B R\} \vDash\left(C_{i} \perp C_{j}\right)$ iff $\left\{\left(A_{1} \equiv \neg B_{1}\right) \vee\left(A_{2} \equiv \neg B_{2}\right) \vee(P \equiv \neg R)\right\}$. 
2. Role level: The Role level mapping between two ontologies can be given by the bridging axioms $\mathrm{BR}_{i j}$, which are relations in the subset of $\Delta^{I_{i}} \times \Delta^{I_{i}} \times \Delta^{I_{j}} \times \Delta^{I_{j}}$. Bridging rules for this can be deduced using both TBox and Abox reasoning. TBox reasoning: DL roles are defined by binary relations, showing the relationships between two concepts. Role $\mathrm{R}(\mathrm{x}, \mathrm{y})$ defines the relationship $\mathrm{R}$ between entities $\mathrm{x}$ and $\mathrm{y}$. Concept $C_{d}$, where $x \in C_{d}$ is the domain concept while $C_{R}$, where $y \in C_{R}$ is the range concept for role R. Using the concept level relation, role level bridging axioms can be deduced as:

a. Equivalence relation: Roles $\mathrm{P}$ and $\mathrm{R}$ in two ontologies can be said to be equivalent if the following holds:

$\{G O, B R\} \vDash P \equiv R \quad$ iff $\quad C_{D}^{P} \equiv C_{D}^{R} \quad$ and $\quad C_{R}^{P} \equiv C_{R}^{R} \quad$ with the bridging rule $B R_{i j} \rightarrow i: P \equiv j: R$

b. Subsumption-Supersumption relation: Roles $\mathrm{P}$ and $\mathrm{R}$ in two ontologies can be inferred to be in a Subsumption-Supersumption relation if the following holds:

$$
\begin{array}{r}
\{G O, B R\} \vDash P \subseteq R \text { iff (i) }\left\{\left(C_{D}^{p} \equiv C_{D}^{R}\right) \wedge\left(C_{R}^{P} \subseteq C_{R}^{R}\right)\right\} \vee \\
\text { (ii) }\left\{\left(C_{D}^{p} \subseteq C_{D}^{R}\right) \wedge\left(C_{R}^{P} \equiv C_{R}^{R}\right)\right\} \vee \\
\text { (iii) }\left\{\left(C_{D}^{p} \subseteq C_{D}^{R}\right) \wedge\left(C_{R}^{P} \subseteq C_{R}^{R}\right)\right\}
\end{array}
$$

With the bridging axiom $B R_{i j} \rightarrow i: P \subseteq j: R$

c. Overlapping relation: Two roles $\mathrm{P}$ and $\mathrm{R}$ are in an overlapping relationship if the following holds:

$\{G O, B R\} \vDash(P \cap R)$ iff $\left\{\left(C_{D}^{P} \wedge C_{D}^{R}\right) \wedge\left(C_{R}^{P} \wedge C_{R}^{R}\right)\right\}$ with the bridging axiom $B R_{i j} \rightarrow i: P \cap j: R$.

d. Disjoint relation: Two roles are said to be in a disjoint relationship if the following holds:

$\{G O, B R\} \vDash(P \perp R)$ iff $\left\{\left(C_{D}^{P} \equiv \neg C_{D}^{R}\right) \vee\left(C_{R}^{P} \equiv \neg C_{R}^{R}\right)\right\}$

Abox reasoning: Using the instances of database Abox reasoning can be used to determine the bridging axioms between the roles of two ontologies. The process is as follows 
a. Equivalence relation: Roles $\mathrm{P}$ and $\mathrm{R}$ are said to be equivalent if the following holds:

$\{G O, B R\} \vDash P \equiv R$ iff $\forall x, y P(x, y) \leftrightarrow R(x, y)$

b. Subsumption-Supersumption relation: Roles $\mathrm{P}$ and $\mathrm{R}$ can be inferred Subsumption-Supersumption relationship if the following holds:

$\{G O, B R\} \vDash P \subseteq R$ iff (i) $\forall x, y, P(x, y) \rightarrow R(x, y)$ and

(ii) $\exists x, y, R(x, y) \wedge \neg P(x, y)$

c. Overlapping relation: Two concepts A and B are in an overlapping relationship if the following holds:

$\{G O, B R\} \vDash(P \cap R)$ iff $\exists x, y P(x, y) \wedge R(x, y)$

d. Disjoint relation: Two concepts are said to be in a disjoint relation if the following holds:

$\{G O, B R\} \vDash(P \perp R)$ iff $\forall x, y P(x, y) \leftrightarrow \neg R(x, y)$

3. Concept vs. Role: In ontology development, it is possible that a concept in one ontology is described as a role in another ontology (Ghidini and Serafini, 2006). Concept to role mapping between two ontologies is achieved by the bridging axiom $B R_{i j}$, finding a relation from $\Delta^{I_{i}}$ to $\Delta^{I_{j}} \times \Delta^{I_{j}}$ a subset of $\Delta^{I_{i}} \times \Delta^{I_{j}} \times \Delta^{I_{j}}$.

TBox Reasoning: Assuming concepts $C_{i}$ and $C_{i^{\prime}}$ in an ontology $i$, if a notion of $C_{i}$ (as concept) and $R_{j}$ (as role) in ontologies $i$ and $j$ are interrelated if:

a. Equivalence relation: Concept $C_{i}$ and Role $R_{j}$ are said to be equivalent if the following holds:

$\{G O, B R\} \vDash C \equiv R$ iff $\left\{\left(C_{i} \equiv C_{D}^{R}\right) \wedge\left(C_{i^{\prime}} \equiv C_{R}^{R}\right)\right\}$, where $C_{D}^{R}$ and $C_{R}^{R}$ are domain and range concepts of role $R_{j}$ and $C_{i^{\prime}}$ is the related concept of domain in ontology $j$ to ontology $i$.

b. Subsumption-Supersumption relation: Concept $C_{j}$ and Role $R_{j}$ can be inferred to be in a Subsumption-Supersumption relationship if the following holds: $\{G O, B R\} \vDash C_{i} \subseteq R_{j}$ iff (i) $\left\{\left(C_{i^{\prime}} \subseteq C_{D}^{R_{j}}\right) \wedge\left(C_{i} \subseteq C_{R}^{R_{j}}\right)\right\} \vee$

(ii) $\left\{\left(C_{i^{\prime}} \subseteq C_{D}^{R_{j}}\right) \wedge\left(C_{i} \equiv C_{R}^{R_{j}}\right)\right\} \vee$

(iii) $\left\{\left(\mathrm{C}_{i^{\prime}} \subseteq C_{D}^{R_{j}}\right) \wedge\left(C_{i} \subseteq C_{R}^{R_{j}}\right)\right\}$ 
c. Overlapping relation: Concept $C_{i}$ and role $R_{j}$ are in an overlapping relationship if the following holds: $\{M O, B R\} \vDash\left(C_{i} \cap R_{j}\right)$ iff $\left\{\left(C_{i^{\prime}} \wedge C_{D}^{R_{j}}\right) \wedge\left(C_{i} \wedge C_{R}^{R_{j}}\right)\right\}$

d. Disjoint relation: Concept $C_{i}$ and $R_{j}$ are said to be in a disjoint relationship if the following holds:

$\{G O, B R\} \vDash\left(C_{i} \perp R_{j}\right)$ iff $\left\{\left(C_{i^{\prime}} \equiv \neg C_{D}^{R_{j}}\right) \vee\left(C_{i} \equiv \neg C_{R}^{R_{j}}\right)\right\}$

Abox Reasoning: Using the instances from database, Abox reasoning can be used to determine the bridging axioms between concepts and roles of two ontologies. The process is as follows:

a. Equivalence relation: Concept $C_{i}$ and Role $R_{j}$ are said to be equivalent if the following holds:

$\{M O, B R\} \vDash A \equiv R_{j}$ iff $\forall x, y C_{i^{\prime}}(x) \wedge C_{i}(y) \leftrightarrow R_{j}(x, y)$.

b. Subsumption-Supersumption relation: Concept $C_{i}$ and $R_{j}$ can be inferred as a Subsumption-Supersumption relationship if the following holds: $\{M O, B R\} \vDash C_{i} \subseteq R_{J}$ iff (i) $\forall x, y, C_{i^{\prime}}(x) \wedge C_{i}(y) \rightarrow R_{j}(x, y)$ and (ii) $\exists x, y, R_{j}(x, y) \wedge\left\{\neg\left(C_{i^{\prime}}(x) \wedge C_{i}(y)\right)\right\}$

Similar line of argument and equation can be built for $\{M O, B R\} \vDash C_{i} \supseteq R_{J}$.

c. Overlapping relation: Concepts $C_{i}$ and Role $R_{j}$ are in an overlapping relationship if the following holds: $\{M O, B R\} \vDash\left(C_{i} \cap R_{j}\right)$ iff $\exists x, y C_{i^{\prime}}(x) \wedge C_{i}(y) \wedge R_{j}(x, y)$

d. Disjoint relation: Two concepts are said to be in a disjoint relationship if the following holds:

$\{M O, B R\} \vDash\left(C_{i} \perp R_{j}\right)$ iff $\forall x, y C_{i^{\prime}}(x) \wedge C_{i}(y) \leftrightarrow \neg R_{j}(x, y)$

So far, this mapping approach has considered the one to one mapping between concepts, roles and concept-role. However, it is highly likely that a concept or role in one ontology is equivalent to subclass - superclass of a combination of concepts and roles in another ontologies as different ontologies may use different levels of granularity for their definitions. Such relationship or bridging axioms can be determined by one to many (or inversely by many to one) mappings. These can be done between a concept in one ontology to its sub-concepts in another ontology or between a 
role in one ontology and its sub-roles in another ontology or a mixture of both. The process of deducing bridging axioms or relationship is as follows:

a. Concept vs. concepts: Suppose a concept A in one ontology has many subsumption relations with concepts $\mathrm{B}_{1}, \mathrm{~B}_{2}, \mathrm{~B}_{3}, \ldots \mathrm{B}_{\mathrm{n}}$ in another ontology. The equivalence relations between them can be found by both Tbox and Abox reasoning. The process of Tbox reasoning is to analyse relation at an atomic level as mentioned in the concept level mapping section and this can be given by the following equation: (Assuming $A \equiv A_{1} \cup A_{2} \cup \ldots \ldots \cup A_{m}$ ) $\left\{M O, B R_{i j}\right\} \vDash\left(A \equiv B_{1} \cup \ldots \ldots \ldots B_{m}\right)$ iff $, A_{1} \equiv B_{1}, \ldots \ldots \ldots . . ., A_{m} \equiv B_{m}$

Through Abox reasoning the equivalence relation can be established by the following equation:

$\{M O, B R\} \vDash\left(A \equiv B_{1} \cup B_{2} \cup \ldots \ldots \cup B_{m}\right)$ iff $\forall x, A(x) \leftrightarrow B_{1}(x) \vee B_{2}(x) \vee \ldots \ldots \ldots . . \vee B_{m}(x)$.

Similar lines of argument and equations (TBox and Abox) can be given for subsumption-supersumption and other relations as previously described.

b. Role vs roles: If a role $P_{i}$ in one ontology has many sub role properties $R_{j}^{1}, R_{j}^{2}, \ldots \ldots \ldots$ in another ontology. The equivalence relation between them can be deduced using Tbox and Abox reasoning as follows:

TBox Reasoning: $\left\{G O, B R_{i j}\right\} \vDash P_{i} \subseteq \bigcup_{k} R_{j}^{k}$ iff (i) $\left\{\left(C_{D}^{p} \equiv \bigcup_{i} C_{D}^{R_{j}^{k}}\right) \wedge\left(C_{R}^{P} \equiv \bigcup_{i} C_{R}^{R_{j}^{k}}\right)\right\}$

Where, $C_{D}^{R_{j}^{k}}$ and $C_{R}^{R_{j}^{k}}$ are the domain and range of role $R_{j}^{k}, C_{D}^{P}$ and $C_{D}^{R}$ are the range and domain of concepts of role $P_{i \text {. }}$

Abox Reasoning:

$\{M O, B R\} \vDash\left(P \equiv R_{1} \cup R_{2} \cup \ldots \ldots \cup R_{m}\right)$ iff $\forall x, y P(x, y) \leftrightarrow R_{1}(x, y) \vee R_{2}(x, y) \vee \ldots \ldots \ldots . . . R_{m}(x, y)$

Similar lines of argument and equations (TBox and Abox) can be given for subsumption-supersumption and other relation as previously described .

In this mapping approach, concepts and roles are compared at the atomic level which will resolve the ontology mapping conflicts described in the introduction section. Atomic level comparison between the concepts will resolve the subclass and classcoverage conflicts as the atomic level relationships are validated using human 
mediation (TBox/Abox input). As for the class-role conflicts, role conflicts and role attribute conflicts, these can be resolved by comparison of the domain and range of roles at the atomic level (similar to class conflicts).Synonymy and polysemy conflicts, which occur due to linguistic characteristics, can be resolved by first using Wordnet (an API for finding the relationships between the words, http://wordnet.princeton.edu/ ) and then by comparing them at the atomic level. The next section describes the implementation method.

\section{Implementation Method and Example:}

The process of implementation or deducing bridging axioms between ontologies is summarized in the figure (3). The first step starts with identifying concepts, roles (or properties) in ontologies using an ontology API (e.g. Jena) and providing them with different namespaces. The second step identifies the lexical similarity using Wordnet and the final step uses the DL reasoning (Abox and Tbox) to deduce the bridging axioms. In this process, a global ontology is formed by incorporating all the entities of all the ontologies for mapping and identifying all their possible relationships.

$$
<<\text { Insert Figure } 3 \text { about here }>>
$$

In addition to using Wordnet, the process of finding lexical similarities (i.e. Synonyms, hyponyms etc.) can be enhanced by providing user interfaces to help the user identify similar words for any concepts and roles. As mentioned in the previous section, Tbox reasoning can be used for concepts, and therefore their relationships, can be deduced if the relationships between atomic concepts of different ontologies can be provided by experts or users. Alternatively, Abox reasoning can be applied if the ontologies provide the same Abox assertions.

In the final step, DL reasoner is used to find the relationships between the entities (concepts and roles) and to establish the bridging rules between the ontologies. The next section describes the Description Logic (DL) perspective of ontology mapping. 


\subsection{Example:}

To illustrate the whole process, two basic ontologies for manufacturing and marketing enterprises have been developed (figure 4) and mapped to assist collaboration between the enterprises. The ontologies were developed using Protégé and exported as owl files to access java APIs. The next step starts by using reasoners (Pellet and SPARQLDL Java API) to find the relationships. The relationships between atomic concepts such as EnterpriseA: Product $=$ EnterpriseB: Product, were provided as a starting point for this research example. The remaining lexically similar entities were compared in the reasoner and the relationships obtained are as follows:

$$
<<\text { Insert Figure } 4 \text { about here }>>
$$

\section{Class vs Class:}

As shown in the figure (5). Concepts Repair and Replacement in ontology A are equivalent to concepts Rectify and Renewal respectively in ontology B (TBox input). The inference that A:Service $\equiv B$ : Service is as follows:

A: Service $\equiv A:$ Repair $\cup$ A: Replacement

$B:$ Service $\equiv B:$ Rectify $\cup B:$ Renewal

A:Repair $\equiv B:$ Rectify

A: Replacement $\equiv B:$ Renewal

Using equation (1.2),(1.3) and (1.4) the equation (1.1) becomes

A: Service $\equiv B:$ Rectify $\cup B:$ Renewal

From equation (1.5), the inference, $A$ : Service $\equiv B$ : Service can be established.

$<<$ Insert Figure 5 about here $>>$

As shown in the figure (6), the concept Material_Property in ontology A does not have an equivalent concept in ontology B. However, subconcepts of Material_Property do have equivalence relations and the corresponding relations are established as follows: 
Using equation (1.6), (1.7) and (1.8), the infered correspondence is A:Material_Property $\equiv B:$ Material $\cup B:$ Strength

$$
<<\text { Insert Figure } 6 \text { about here }>>
$$

In many cases, as described in the previous section, hetrogenity occurs in the ontology mapping due to sub-class conflicts. As shown in the figure (7), the concept Dimension in ontology A has six sub-concepts, where as in ontology B the concept Dimension has no sub concepts. Given in the TBox similarity as :

\section{A:Dimension $\equiv A:$ Bore $\cup A:$ Breadth $\cup A:$ Depth $\cup A:$ Height $\cup A:$ Length $\cup A:$ Radius}

A: Dimension $\supseteq B:$ Dimension

The following is infered:

$B:$ Dimension $\subseteq$ A: Bore $\cup$ A $:$ Breadth $\cup$ A: Depth $\cup$ A $:$ Height $\cup A:$ Length $\cup$ A: Radius ..

$<<$ Insert Figure 7 about here $>>$

2. Property vs Property

Implementation of the proposed methods, as described in the previous section, to find correspondence between properties or roles, checks the correspondence between the range and domain of roles. As shown in the figure (8), the correspondence between role hasDimension of ontology A and B is inferred as follows:
A: hasDimension $(A:$ Product, A: Bore $\cup$ A: Breadth $\cup$ A:Depth $\cup$ A: Height $\cup$ $A:$ Length $\cup A:$ Radius)

Here the first part in the brackets states the domain and second part states the range of the role hasDimension 
Using the result (1.11), it can be rephrased as:

A: hasDimension(A: Product, A: Dimension)

Similarly,

B : hasDimension(B: Product, B : Dimension)

it is clear that $A:$ Product $\equiv B:$ Product and $A:$ Dimension $\supseteq B:$ Dimension. This gives the inference, $A$ : hasDimension $\supseteq B$ : hasDimension .

$<<$ Insert Figure 8 about here $>>$

3. Class vs Property

As mentioned in the previous section, a concept in one ontology can be described as role in another ontology. In order to determine the correspondence between these two, analysis of concepts and range and domain of role must be examined. As shown in the figure (9), lexical similarity is obtained between Troubleshooting and hasTroubleshooting. Their correspondence is inferred as follows:

$B:$ hasTrobleshooting( $B:$ Product, $B:$ Failure $\cup B:$ Malfunctioning)

A: Product $\equiv B:$ Product

A:TroubleShooting $\equiv B:$ Failure $\cup B:$ Malfunctioning

Using equation (3.1),(3.2) and (3.3), the following is inferred:

B: hasTroubleShooting (A: Product, A:Troubleshooting)

Equation (3.1) and equation (3.4) correlates the correspondence according to the class-property similarity condition and the following can therefore be inferred:

A:Troubleshooting $\equiv B$ : hasTroubleshooting

$<<$ Insert Figure 9 about here $>>$

\section{Conclusion and Future Research:}

This paper presented a DL based axiom derivation methodology that exploits synonyms and axiomatic matching to find the correspondence between the two ontologies in the 
form of bridging axioms without changing the entities name and by forming a global ontology. This process will help to achieve interoperability between the ontologies of different enterprises forming a VE and will facilitate the sharing of data, information and knowledge with correct semantic and intention. The bridging axioms discussed in this paper will help to achieve the correct inference. As presented in the introduction section, the bridging axiom 01: hasBore $\subseteq 02$ : hasDiameter will allow inference that hasBore $\rightarrow$ hasDiameter but will not allow the inference hasDiameter $\rightarrow$ hasBore. The concept level inconstancies as described in the introduction section could be avoided by the identification of the following axioms through the proposed methodology:

1. $01:$ peration $\equiv(02:$ pperation $\cup 02:$ Transportation $)$

2. 01:Operation $\subseteq 02:$ Operation

3. 01:Transportation $\equiv 02$ : Transportation

From the above equation, equation (i) in the introduction section will change to:

02: Operation $\rightarrow$ 01: Operation and the wrong inference will be avoided. Thus the method proposed in this paper will bring consistency in ontology mapping.

The complexity of this approach has not been considered in the current work, however future research includes the examination of complexity levels of this approach, consideration of more expressive DL languages and development of a query translation mechanism in the global ontology. 


\section{$\underline{\text { References: }}$}

Bouquet, P., Serafini, L., Zanobini, S., Sceffer, S., 2006. Bootstrapping semantics on the web: meaning elicitation from schemas. , 505-512.

Chen, D., Doumeingts, G., Vernadat, F., 2008. Architectures for enterprise integration and interoperability: Past, present and future. Comput. Ind. 59, 647-659.

Chen, R., Bau, C., Yeh, C., 2011. Merging domain ontologies based on the WordNet system and Fuzzy Formal Concept Analysis techniques. Applied Soft Computing 11, 1908-1923.

Chungoora, N., Young, R.I.M., 2008. Semantic Interoperability Requirements for Manufacturing Knowledge Sharing, in Mertins, K., Ruggaber, R., Popplewell, K., Xu, X. (Eds.), Enterprise Interoperability III. Springer London, pp. 411-422.

Doan., A., Madhavan., J., Domingos., P., Halevy ., A., 2004. Ontology Matching: a machine learning approach, Hanbook on Ontologies. Springer-Verlag, Berlin.

Dou, D., McDermott, D.V., 2006. Towards Theory Translation. , Post-Proceedings of the 4th International Workshop on Declarative Agent Languages and Technologies (DALT) (Invited Paper), 16-28.

Euzenat, J., 1994. Brief overview of T-tree: The TROPES taxonomy building tool. Columbus (OH, US). 69-87.

Euzenat, J., Valtchev, P., 2004. Similarity-Based Ontology Alignment in OWL-Lite. , 333337.

Euzenat, J., Shvaiko, P., 2007. Ontology Matching, Springer-Verlag, Berlin.

Giunchiglia, F., Shvaiko, P., Yatskevich, M., 2004. S-Match: an Algorithm and an Implementation of Semantic Matching, in Bussler, C., Davies, J., Fensel, D., Studer, R. (Eds.), The Semantic Web: Research and Applications. Springer Berlin / Heidelberg, pp. 61-75. 
Gruber, T., R., 1995. Toward principles for the design of ontologies used for knowledge sharing? International Journal of Human-Computer Studies 43, 907-928. (Dou and McDermott, 2006)

Hu., W., Cheng., G., Zheng., D., Zhong., X., Qu., Y., 2006. The results of Falcon-AO in the OAEI 2006 campaign. International workshop on Ontology Matching .

Hu, W., Qu, Y., Cheng, G., 2008. Matching large ontologies: A divide-and-conquer approach. Data Knowl. Eng. 67, 140-160.

Jagdev, H.S., Thoben, K., 2001. Anatomy of enterprise collaborations. Production Planning \& Control 12, 437-451.

Kalfoglou, Y., Schorlemmer, M., 2003. IF-Map: An Ontology-Mapping Method Based on Information-Flow Theory, in Spaccapietra, S., March, S., Aberer, K. (Eds.), Journal on Data Semantics I. Springer Berlin / Heidelberg, pp. 98-127.

Martinez, M.T., Fouletier, P., Park, K.H., Favrel, J., 2001. Virtual enterprise - organisation, evolution and control. Int J Prod Econ 74, 225-238.

Massmann., S., Engmann., D., Rahm., E., 2006. COMA ++:results for the ontology alignment contest OAEI 2006. International workshop on Ontology Matching .

Mitra., P., Wiederhold., G., 2002. Resolving Terminological Heterogeneity in Ontologies. Workshop on Ontologies and Semantic Interoperability at the 15th ECAI , 45-50.

Noy, N.F., Musen, M.A., 2003. The PROMPT suite: interactive tools for ontology merging and mapping. International Journal of Human-Computer Studies 59, 983-1024.

Pollalis, Y.A., Dimitriou, N.K., 2008. Knowledge management in virtual enterprises: A systemic multi-methodology towards the strategic use of information. Int. J. Inf. Manage. $28,305-321$.

Stumme, G., Maedche, A., 2001. FCA-MERGE: Bottom-Up Merging of Ontologies. , 225234.

Wang, H., Liu, J.N.K., 2009. Analysis of Semantic Heterogeneity Using a New Ontological Structure Based on Description Logics. Sixth International conference on Fuzzy Systems and Knowledge Discovery, FKSD '09. 